\title{
Anti-Inflammatory Activity of Sanjie Zhentong Capsule Assessed By Network Pharmacology Analysis of Adenomyosis Treatment
}

This article was published in the following Dove Press journal: Drug Design, Development and Therapy

\author{
Li Du (D) \\ De-Hui Du ${ }^{2}$ \\ Biao Chen ${ }^{2}$ \\ Yue Ding ${ }^{3}$ \\ Tong Zhang ${ }^{3}$ \\ Wei Xiaol,4
}

'Institute of Chinese Materia Medica, Shanghai University of Traditional Chinese Medicine, Shanghai, People's Republic of China; ${ }^{2}$ Shanghai Key Laboratory of Trustworthy Computing and Software Engineering Institute, East China Normal University, Shanghai, People's Republic of China; ${ }^{3}$ Experiment Center for Teaching and Learning, Shanghai University of Traditional Chinese Medicine, Shanghai, People's Republic of China; ${ }^{4}$ jiangsu Kanion Pharmaceutical Co., Ltd, Jiangsu, People's Republic of China
Correspondence: Tong Zhang

Experiment Center for Teaching and Learning, Shanghai University of

Traditional Chinese Medicine, I 200 Cailun Road, Shanghai 201203, People's Republic of China

Tel +86 02I-5132-3175

Email zhangtdmj@hotmail.com

Wei Xiao

Jiangsu Kanion Pharmaceutical Co., Ltd, Jiangning Industrial City, Economic and

Technological Development Zone, Jiangsu, Lianyungang 22200 I, People's Republic of China

Tel +8605I8-85521956

Email xw-kanion@I63.com
Background: Sanjie Zhentong capsule (SZC) offers excellent effect in treating adenomyosis (AM), which is a common and difficult gynecological disease in the clinic. However, the systematic analysis of its mechanism has not been carried out yet and further studies are needed to reveal the role of SZC.

Methods: A systematic network pharmacology analysis was conducted by integrating construction of SZC compound database and AM target database, prediction of potential active compounds and targets by molecular docking combined with compound-target prediction graph (CTPG), protein-protein interaction (PPI) analysis, Gene Ontology (GO) and Kyoto Encyclopedia of Genes and Genomes (KEGG) pathway analysis. Then, the anti-inflammation experiments in vitro were performed by investigating SZC and the representative compounds regulating nitric oxide (NO), interleukin-6 (IL-6), and interleukin-10 (IL-10).

Results: Our findings show that SZC mainly treated AM by stimulating 28 core targets through 30 key potential active compounds, and affecting 4 crucial pathways. The treatment was associated with inflammation reaction, hormone regulation, cell adhesion, proliferation, and angiogenesis. Additionally, SZC achieved the anti-inflammatory activity by the cooperation of the compounds through inhibiting NO and IL-6, both promoting and inhibiting IL-10. Conclusion: This study investigated the anti-inflammatory activity of SZC based on a systematic analysis of SZC remedying AM, which was revealed to be one of the essential mechanisms. These findings will provide valuable guidance for further research of the SZC treatment of AM, and help improve the comprehension of SZC pharmacological basis as well as AM pathogenesis.

Keywords: Sanjie Zhentong capsule, adenomyosis, network pharmacology, molecular docking, compound-target prediction graph, anti-inflammation

\section{Introduction}

Adenomyosis (AM), a common difficult gynecological disease, occurs in 6-10\% women of childbearing age, the rate increases to $60 \%$ in infertile women. With the discriminative pathological characteristics of benign infiltration and growth of the endometrium into the myometrium, the ectopic endometrium of the AM patients changes the menstrual cycle (includes bleeding, shedding, and invasion) and results in the proliferation of muscle cells, a thickened myometrium, and enlarged uterine volume. ${ }^{1}$ Although the pathogenesis is still unclear, uterus basement membrane invagination caused by endometrial tissue damage and repair, and embryonic stem cell residue and differentiation, are the two existing theories of AM at present. ${ }^{2}$ The 
former considered that AM is mainly associated with high hormone levels, ${ }^{3}$ tissue damage, and repair, ${ }^{4}$ the latter believed that AM is caused by an embryonic remnant or adult stem cell differentiation. ${ }^{5}$ It has also been revealed that once AM is formed, the critical factors for its evolution and development are invasion and migration. ${ }^{6}$ On the other hand, from the perspective of traditional Chinese medicine (TCM), AM belongs to the same category as "dysmenorrhea" and "various syndromes before and after menstruation", with lesions in the uterus and Chong ren, stagnant blood, qi, and body fluid, and dampness accumulated by phlegm as the pathogenesis. ${ }^{7}$ Since hormones and nonsteroidal anti-inflammatory drugs are generally applied in the treatment of AM, which put a heavy load on patients due to the high cost, high recurrence rate, and severe side effects, the application of TCM is gradually showing its unique advantages. Definitive curative effects have been achieved through TCM therapeutics, for instance, patients with AM treated with Xuanyu Tongjing decoction achieved a recovery rate of $90.9 \%$, the treatment improving dysmenorrhea symptoms, relieving pain, and causing few adverse reactions. ${ }^{8}$ The total effective rate reached $96.8 \%$ when the patients had been treated with Jiawei Siwu decoction, and this treatment exhibiting a significant efficacy difference compared with that provided by a chemical medicine group. ${ }^{9}$

Sanjie Zhentong capsule (SZC) is a traditionally prescribed as Chinese medicine that is made by the original powder of four natural drugs: Longxuejie, Sanqi, Zhebeimu, and Yiyiren. It has been used for secondary dysmenorrhea, irregular menstruation, pelvic mass and infertility caused by endometriosis (phlegm and blood stasis mixed with qi stagnation syndrome) in the clinic. SZC is beneficial in treating ovarian cysts, ${ }^{10}$ reducing the development of endometriosis ${ }^{11}$ and achieving superior inhibition of the focused growth of endometriosis in rats. ${ }^{12}$ In addition, SZC has also been used in the clinical application of AM. Researchers have found that SZC could dramatically reduce the operation rate of AM by improving the pregnancy rate and clinical symptoms in AM patients who experience infertility, ${ }^{13}$ and by treating dysmenorrhea caused by AM. ${ }^{14}$ It has also been presented that SZC treatment of AM might be related to the significant decrease of CA125, TNF- $a$, and PGF-2a in the serum. ${ }^{15}$ Nevertheless, the complex pathogenesis of AM, as well as the multiplex mechanism of SZC remain unclear, the principles and mechanisms by which SZC is effective for treating AM are needed to be uncovered.
In recent years, increasing attention has been paid on the holistic philosophy of TCM, the mode of drug discovery design has shifted from single-target to network-target and multiple-component-therapeutics with a systematic view. ${ }^{16}$ With the updates of bioinformatics and computer technology, network pharmacology has been developed rapidly to become a credible modern approach for predicting the potential active compounds of TCM and available targets of disease, and exploring mechanisms. For example, Shi has proposed a systematic network pharmacology approach, identified 29 compounds acting on 16 pathways (regulated by 32 targets), and revealed the blood enriching mechanism of Danggui buxue decoction. ${ }^{17}$ Yang applied network pharmacology to investigate the antimetastasis mechanism of Oldenlandia Diffusa in breast cancer and found that 12 compounds and 85 targets were associated with the treatment. ${ }^{18}$ However, the systematic analysis of SZC treatment of AM has not yet been carried out. Herein, we conducted a network pharmacological analysis of SZC treatment of AM by proposing an integrated approach. Furthermore, experiments in vitro were performed to observe the antiinflammation activity of SZC. The workflow is shown in Supplementary Figure S1.

\section{Materials and Methods}

\section{SZC Compound Database Construction}

We applied Web crawler technology to automatically pick up compound information from the TCMSP database (http://lsp.nwu.edu.cn/tcmspsearch.php), and then stored it in the SZC compound database. Longxuejie is not in the TCM databases, wide-scale text mining of PubMed (https://www.ncbi.nlm.nih.gov/pubmed/) and CNKI (http://www.cnki.net/) databases was performed. The 3D structure files with SDF format of the compounds were crawled and downloaded from the NCBI compound database according to their retrieved names and PubChem identifiers from the database of chemicals (https://pubchem.ncbi.nlm.nih.gov/). Besides, we used the selenium crawler framework, an automated testing framework that is ideal for crawling dynamic web pages, making it easy to crawl data quickly and precisely on objective sites. Throughout the process, we adopted python language to design crawlers which help realize automatically acquiring and recognizing information of compound data. 


\section{Target Database Construction of AM}

To prepare the protein information for molecular docking, we acquired information of targets from the NCBI gene database by searching the name of disease "adenomyosis" and stored it into the AM target database. Then, corresponding protein information was searched from UniProt (https://www.uniprot.org/) and stored in the AM target database using the web crawler technology earlier described. Notably, when performing protein screening in UniProt, proteins of homo species with a lower resolution were selected.

\section{Prediction of Potentially Active Compounds and Targets}

To predict the potential active compounds and targets, we adopted molecular docking which has been widely used to describe the strength of binding interactions between molecules. The prediction process consists of two phases. First, System Dock Web Site (http://systemsdock.unit.oist. $\mathrm{jp} / \mathrm{iddp} /$ home), which is a very popular web server in network pharmacology-based prediction, was linked to perform the molecular docking process with the help of automatic link docking algorithms. We have implemented the algorithms to implement the prediction. Generally, compound-target pairs with the score $\geq 4.25$ are considered to have particular binding activity. The score $\geq 5.0$ indicates a greater binding activity and score $\geq 7.0$ indicates a stronger binding interaction between compound and target. ${ }^{19}$ Second, a Compound-Target Prediction Graph (CTPG) is proposed to model the predicting of the potential active compounds. The targets was built based on a directed graph with weighted edges, which manifests the cohesion of the network ${ }^{20}$ and illustrates the cohesion degree of the elements with the clustering coefficient. ${ }^{21}$ In $C T P G=(V, E, W), V$ denotes a finite set of vertexes, which represents the compounds or targets. $E$ denotes the directed edge including the output edge and the input edge between compounds and targets. $W$ denotes the total score of a particular compound or target. After calculating the $W$ value and the number of targets linked to a compound as well as the number of compounds linked to a target, we selected the compound or target with superior $W$ value and a bigger linked number as the potentially active one. Therefore, the CTPG was effective to explain the degree of strength that they contribute to the mechanism by analyzing the distribution of compounds and targets. The details of adopting CTPG can be found in our experiments.

\section{Protein-Protein Interaction (PPI) Analysis}

The interaction among proteins of the potential active targets was analyzed furtherly. STRING database (https:// STRING-db.org/) was adopted to analyze the PPI. The protein data of potential targets was imported into STRING, the organism was set as homo sapiens, and then the result of PPI was visualized with Cytoscape v3.7.1.

\section{Go and KEGG Enrichment Analysis}

To understand the enrichment of the acquired potential target proteins and differential genes in biological functions and pathological pathways, cellular localization, GO annotation analysis and the KEGG pathway enrichment analysis of the achieved targets were performed. The terms with P-value $<0.05$ were screened for the main functional annotation and significant pathways clustering. The less correlated ones were removed. ClueGO and CluePedia plugins of Cytoscape v3.7.1 were utilized in the GO enrichment analysis and KEGG pathway enrichment analysis.

\section{Network Construction and Analysis}

To intuitively understand the mechanisms of SZC treatment on AM, both the compound-target network and target-pathway network were constructed. The graphs of the networks were generated and visualized using Cytoscape v3.7.1. The formation of the compound-target network was based on the potential active compounds and the corresponding targets, obtained by docking and CTPG analysis. The target-pathway network was built by connecting targets to the signaling pathways. In the network graphs, nodes represent compounds, targets, or signaling pathways, and edges indicate the interactions of compound-target or target-pathway. The degree of a node was determined by the number of edges connected with it.

\section{Anti-Inflammatory Activity of SZC in vitro \\ Reagents}

Ginsenoside Rg1 (HPLC $\geq 98 \%$ ), ginsenoside Rb1 (HPLC $\geq 98 \%$ ), notoginsenoside R1 (HPLC $\geq 98 \%$ ), ginsenoside Rd (HPLC $\geq 98 \%$ ), resveratrol (HPLC $\geq 98 \%$ ), pterostilbene (HPLC $\geq 98 \%$ ), 7.4'-dihydroxyflavone (HPLC $\geq$ 98\%), loureirin A (HPLC $\geq 98 \%$ ), loureirin B (HPLC $\geq$ 98\%) and peiminine (HPLC $\geq 98 \%$ ) were obtained from the Standardization Research Center of TCM (Shanghai, 
China). The concentration of DMSO was $<0.1 \%$ in this study. SZC was obtained from Kanion Pharmaceutical Co., Ltd. (Jiangsu, China). The compounds were stable under the conditions used in the study. All chemical structures are shown in Supplementary Figure S2. Cell Counting Kit8 (CCK-8) was obtained from Beyotime (Shanghai, China), fetal bovine serum (FBS), dulbecco's modified Eagle's medium (DMEM) and penicillin/streptomycin were obtained from Gibco Life Technologies (Waltham, MA, USA), lipopolysaccharide (LPS), sulfanilamide and N-1-naphthyl ethylenediamine hydrochloride were obtained from Sigma-Aldrich LLC (Darmstadt, Germany).

\section{Cell Culture}

RAW264.7 cells (purchased from the Chinese Academy of Sciences, Shanghai, China) were cultured in DMEM containing $10 \% \mathrm{FBS}$ and $1 \%$ penicillin/streptomycin at $37^{\circ} \mathrm{C}$ in the cell incubator with a humid atmosphere containing $5 \% \mathrm{CO}_{2}$.

\section{Cell Viability Assay}

Cell viability test was taken using CCK-8 assay after treatment with the compounds of SZC. In brief, RAW264.7 cells in a logarithmic phase were seeded in a 96-well plate at a density of $1 \times 10^{4}$ cells in the absence or presence of $24 \mathrm{~h}$. After aspirating the medium, the cells were incubated with $10 \% \mathrm{CCK}-8$ for $1 \mathrm{~h}$ and ELISA (MD, USA) was applied to read OD value at $450 \mathrm{~nm}$.

\section{Measurement of Nitric Oxide}

Cells were seeded in a 96-well plate at a density of $5 \times 10^{4}$ cells and incubated as above for $24 \mathrm{~h}, 0.01 \mu \mathrm{g} / \mathrm{mL}$ LPS was added after the cells were incubated with a gradient concentration of SZC for $30 \mathrm{~min}$. To determine the concentration of nitrite in the culture media after $24 \mathrm{~h}$, Griess reagent ( $1 \%$ sulfanilamide, $0.1 \% \mathrm{~N}$-1-naphthyl ethylenediamine hydrochloride) and supernatant at each treatment condition were mixed with the same volume and OD value was read using ELISA reader at $540 \mathrm{~nm}$.

\section{Cytokine Beads Array Assay}

Cells were seeded and treated with SZC and LPS according to the same procedure described above. Supernatant was collected and stored at $-80^{\circ} \mathrm{C}$ until analyzed. Cytokines were measured using the Cytometric Bead Array (CBA) kit (BD Biosciences, CA) according to the manufacturer's protocol. Samples were incubated with capture beads and phycoerythrin detection reagent for 2 $\mathrm{h}$, including standard and test ones, washed with wash buffer and then analyzed using the FACSCalibur flow cytometer with BD CBA software.

\section{Statistical Analysis}

All results were expressed as means $( \pm$ SD). Statistical analysis was performed by Graphpad Prism 7.0, using the one-way analysis of variance (ANOVA) test, followed by Dunnett's multiple comparison post hoc test. $\mathrm{P}<0.05$ was considered to be statistically significant.

\section{Results}

\section{Potential Active Compounds and Targets of SZC}

With the help of selenium and python, the automatic data mining and docking platform linking were realized; combined docking with the compound-target prediction graph (CTPG), active compounds and targets were identified and classified according to their different binding activities, As a result, 224 compounds and 106 targets were stored in the SZC database and target database, respectively. After docking, 105 candidate molecules with docking score above 4.25 (in which, 95 molecules had a docking score above 5 and 38 molecules had a docking score above 7) were screened as compounds with binding activity. We display four compound-protein pairs with a docking score above 7 in Figure 1. The CTPG exhibited the contribution degree of the compounds and targets with a docking score above 4.25 for SZC treating AM. As there was a sharp decline when the total weighted scores came down to 200,78 compounds with docking scores above 4.25 and total weighted scores above 200 that displayed a concentrated distribution were taken for the potential active components of SZC in treating AM. We show the distribution of active compounds in Figure 2A. Additionally, 42 proteins with docking scores above 4.25 and a total weighted score above 400 were screened as the potential targets of AM treated by SZC. We show the distribution of potential targets in Figure 2B. The data of docking and distribution are listed in Supplementary Table S1-S2, respectively.

\section{The Interaction of Proteins}

The 42 potential targets of AM related to SZC obtained above were introduced into the STRING database. as shown in Figure 3. The network involved a total of 42 nodes and 259 edges, in which, neurogenic locus notch homolog protein 1 (NOTCH1), cyclin-D1 (CCND1), estrogen receptor (ESR1), C-C motif chemokine ligand 2 

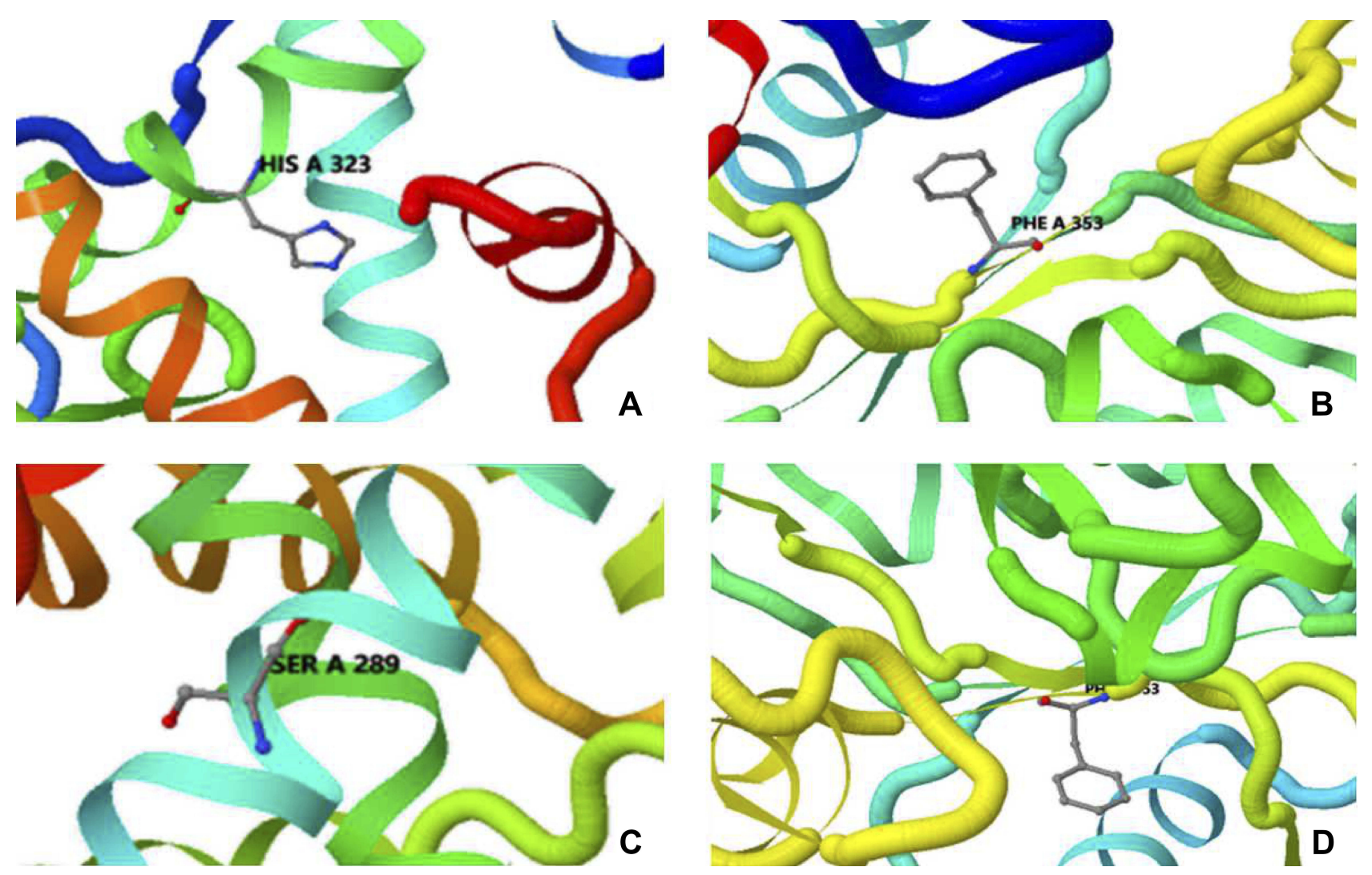

Figure I Compound-target pairs with a docking score above 7. (A) Sitosterol-ESR2, the score is 8.42I, (B) Panaxatriol-CYR6I, the score is 8.409, (C) Sitogluside-PPARG, the score is 8.337, (D) Peiminine-NOS3, the score is 8.20I.

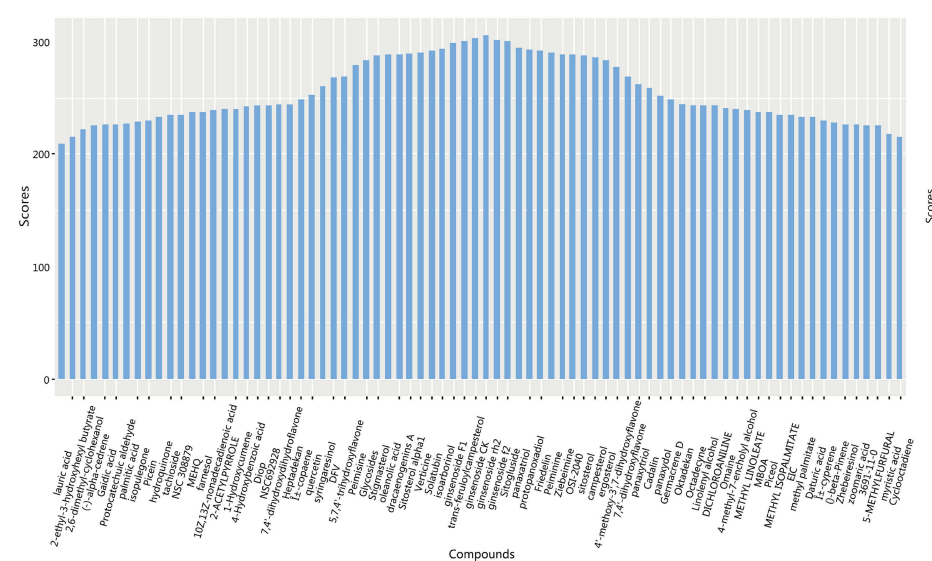

A

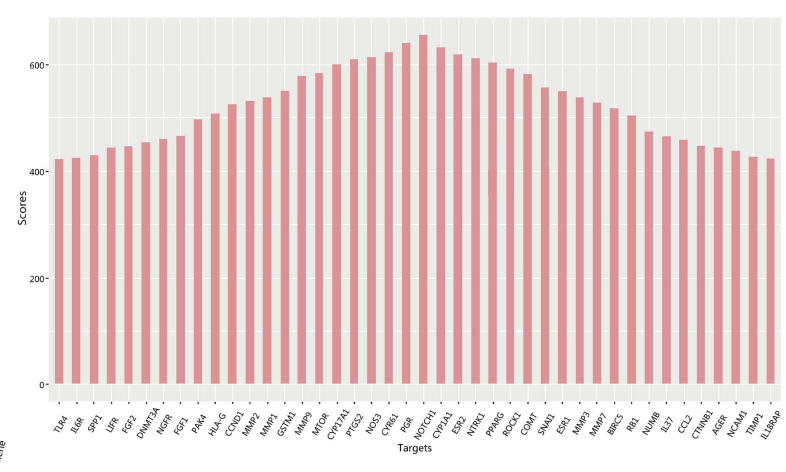

B

Figure 2 CTPG of SZC treating AM. (A) Distribution of potentially active compounds of SZC for treating AM. The y-axis represents total weighted scores of compounds, and the $\mathrm{x}$-axis shows all compounds with total weighted scores higher than 200. (B) Distribution of the potential targets of SZC treated by AM. The $y$-axis represents total weighted scores of targets, and the $\mathrm{x}$-axis shows all targets with total weighted scores higher than 400 .

(CCL2), prostaglandin $\mathrm{G} / \mathrm{H}$ synthase 2 (PTGS2) and matrix metalloproteinase 2/9 (MMP2/9) displayed the biggest size and the darkest color, which interacted with the other targets strongly and predominated the remedy for AM.

\section{GO Annotation and KEGG Enrichment Analysis}

The results of the gene ontology biological process (GOBP) annotation analysis revealed that the potential targets we acquired are closely involved in diverse 


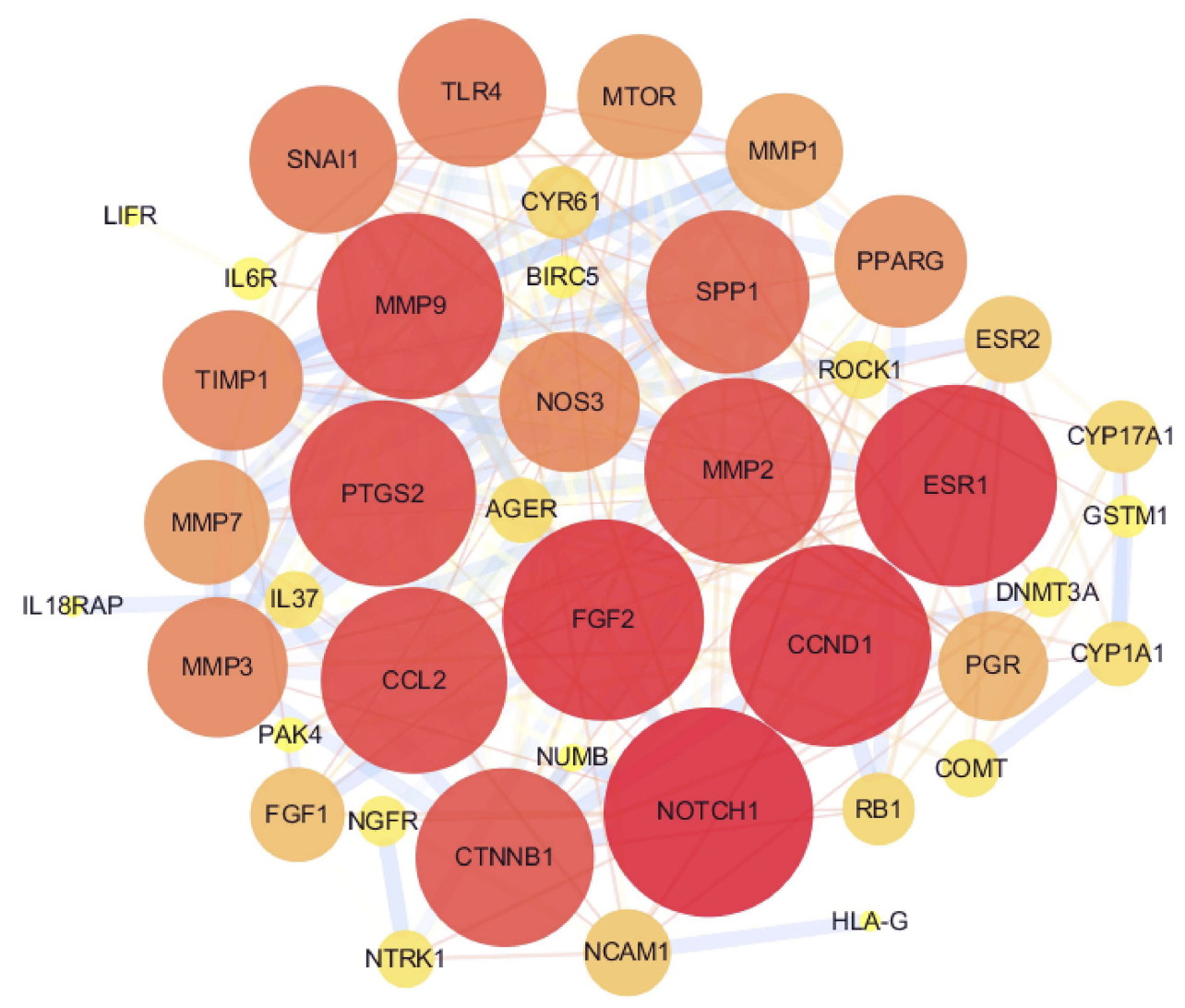

Figure 3 Protein-protein interaction analysis of potential target genes. The nodes represented the proteins, and the edges represented the interrelationships among the proteins. The size and color of the nodes indicate the degree of importance of the targets. The larger the node is, the higher the degree of the importance is, and the degree becomes greater as the color changes from yellow to red. The thickness of the edge indicates the association confidence: the thicker the edge is, the stronger the proteins combined.

biological functions and process concentrated on inflammation, hormones, adhesion, proliferation, and angiogenesis, which are intensely related to the pathogenesis of AM. The top 20 that significantly enriched GOBP terms $(\mathrm{P}<0.05)$ are listed in Figure 4.

\section{Network Construction and Analysis Compound-Target Network Construction and Analysis}

After the CTPG prediction, 30 key potential compounds and 28 targets were screened, showing strong binding activity and major contribution in the group with the docking score above 7 , the compound total weighted score above 200 and the target total weighted score above 400 . The network of key compounds with targets that play the most contributory role in SZC for treating AM is displayed in Figure 5, there were 12 compounds completely from Sanqi; 7 compounds completely from Zhebeimu; 6 compounds completely from Yiyiren; 3 compounds completely from Longxuejie, while, 1 compound from both Sanqi and
Yiyiren; 1 compound from Longxuejie, Zhebeimu, and Yiyiren. Besides, ginsenoside Rh2 (from Sanqi), peiminine (from Zhebeimu), trans-feruloylcampesterol (from Yiyiren) and dracaenogenins A (from Longxuejie), interacted with 27, 27, 23 and 17 targets, respectively; for targets, estrogen receptor (ESR) interacted with 52 compounds, progesterone receptor (PGR) with 28 compounds, PTGS2 with 26 compounds and Nitric oxide synthase 3 (NOS3) with 25 compounds. It significantly indicated the multi-component and multi-target characteristic of SZC in the treatment of AM. Detailed information on the key potential compounds and targets is listed in Tables 1 and 2.

\section{Target-Pathway Network Construction and Analysis}

Pathways of AM treated by SZC were enriched in KEGG, excluding the less relevant ones, 41 pathways $(\mathrm{P}<0.05)$ directly related to AM were obtained, and classified into five main regulation modules, including inflammation reaction, hormone regulation, cell adhesion, proliferation, 


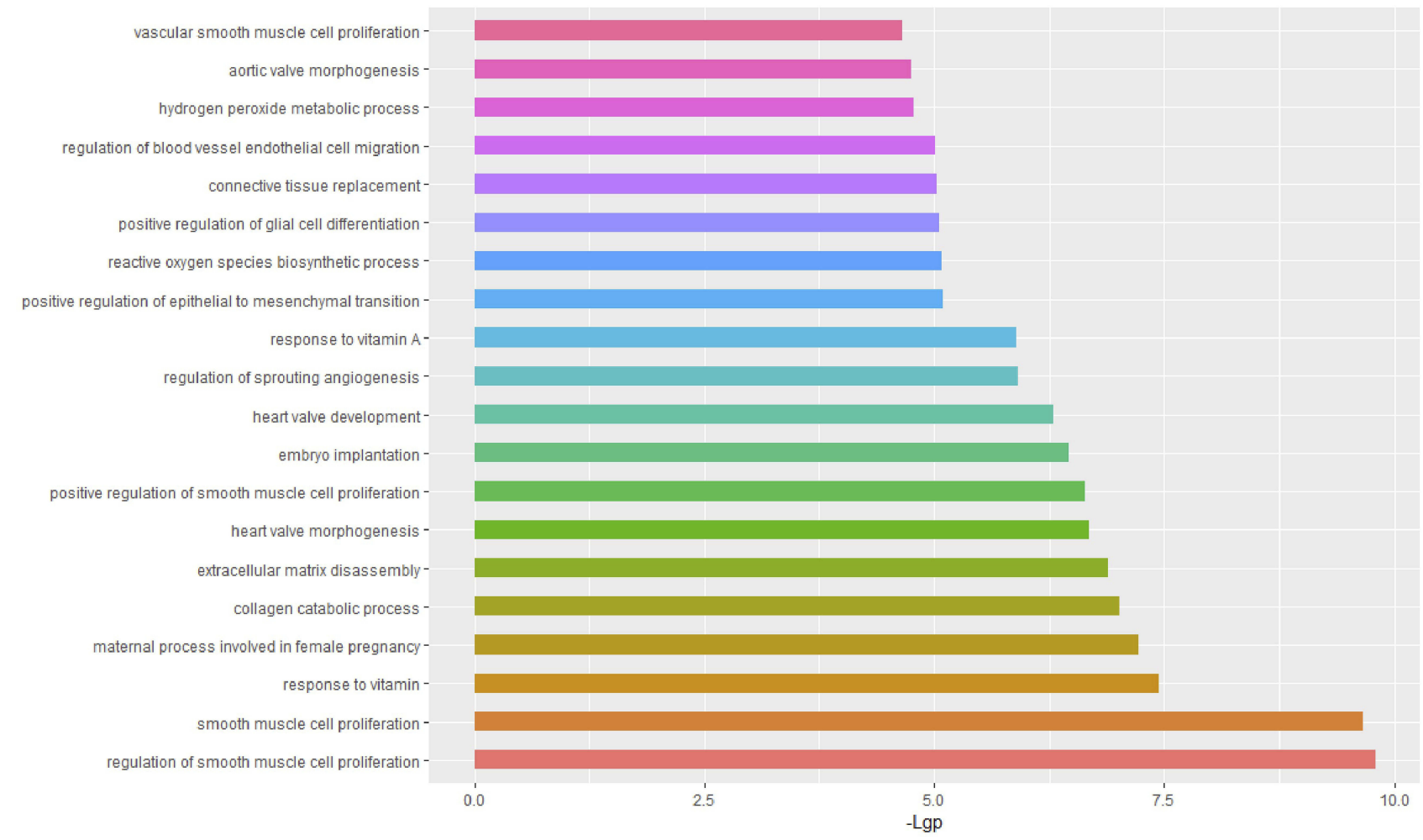

Figure 4 Gene ontology analysis of the potential target genes, the $y$-axis shows the top 20 significantly relevant biological processes enriched with these genes, and the $x$-axis shows the enrichment scores of biological process terms $(p<0.05)$.

and angiogenesis. The target-pathway network graph is presented in Figure 6 including 81 nodes (5 modules, 35 targets, 41 pathways) and 367 edges. We further emphasized on the most representative pathways (endocrine resistance, the PI3K-Akt signaling pathway, focal adhesion, and the NF- $\mathrm{B}$ signaling pathway) and merged them into a confluence map that the PI3K-Akt signaling pathway and the NF- $\mathrm{KB}$ signaling pathway intersected at TLR4; endocrine resistance, the PI3K-Akt signaling pathway, and focal adhesion crossed at mTOR; and CCND1 is the common target protein in the four pathways, as shown in Figure 7. The data of Go terms, pathways and function modules is listed in Supplementary Table S3-S4.

\section{Anti-Inflammatory Activity of SZC in vitro}

Since the inflammatory regulation of SZC was identified to be one of the essential regulation modules in treating AM, we examined the anti-inflammatory activity of SZC as well as ten active compounds of SZC with less cytotoxicity that had been quantified in the previous study. ${ }^{22,23}$ The cytotoxicity test showed that resveratrol, pterostilbene and 7,4'-dihydroxyflavone were cytotoxic to LPS-treated
RAW264.7 cells at a concentration exceeding $20 \mu \mathrm{M}$, while other compounds were safe at concentration even higher than $100 \mu \mathrm{M}$. Then, we set the concentration of pterostilbene and 7,4'-dihydroxyflavone at $20 \mu \mathrm{M}$, loureirin A, loureirin B, notoginsenoside R1, ginsenoside Rg1, ginsenoside $\mathrm{Rb} 1$, ginsenoside $\mathrm{Rd}$, peiminine at $50 \mu \mathrm{M}$, dexamethasone (DXM) at $1 \mu \mathrm{M}$, and SZC at $50 \mu \mathrm{g} / \mathrm{mL}$ as the test concentrations for the next experiments.

After being treated by the selected compounds at certain concentrations, the cell viability was not inhibited compared with the untreated group, which indicated that the compounds at the concentrations were all noncytotoxic to the LPS-treated RAW264.7 cells. The result of the cell viability is presented in Figure 8A.

\section{The NO Synthesis Inhibition of SZC}

As shown in Figure 8B, pterostilbene and SZC exhibited significantly stronger inhibition of NO than DXM $(\mathrm{P}<$ 0.001); resveratrol and ginsenoside $\mathrm{Rd}$ inhibited NO with no significant difference versus DXM, loureirin A and loureirin $\mathrm{B}$ exhibited weaker inhibition of $\mathrm{NO}$ with a significant difference versus DXM $(\mathrm{P}<0.05)$; while 7,4'dihydroxyflavone, notoginsenoside R1, ginsenoside Rg1, ginsenoside $\mathrm{Rb} 1$, and peiminine exhibited significantly 


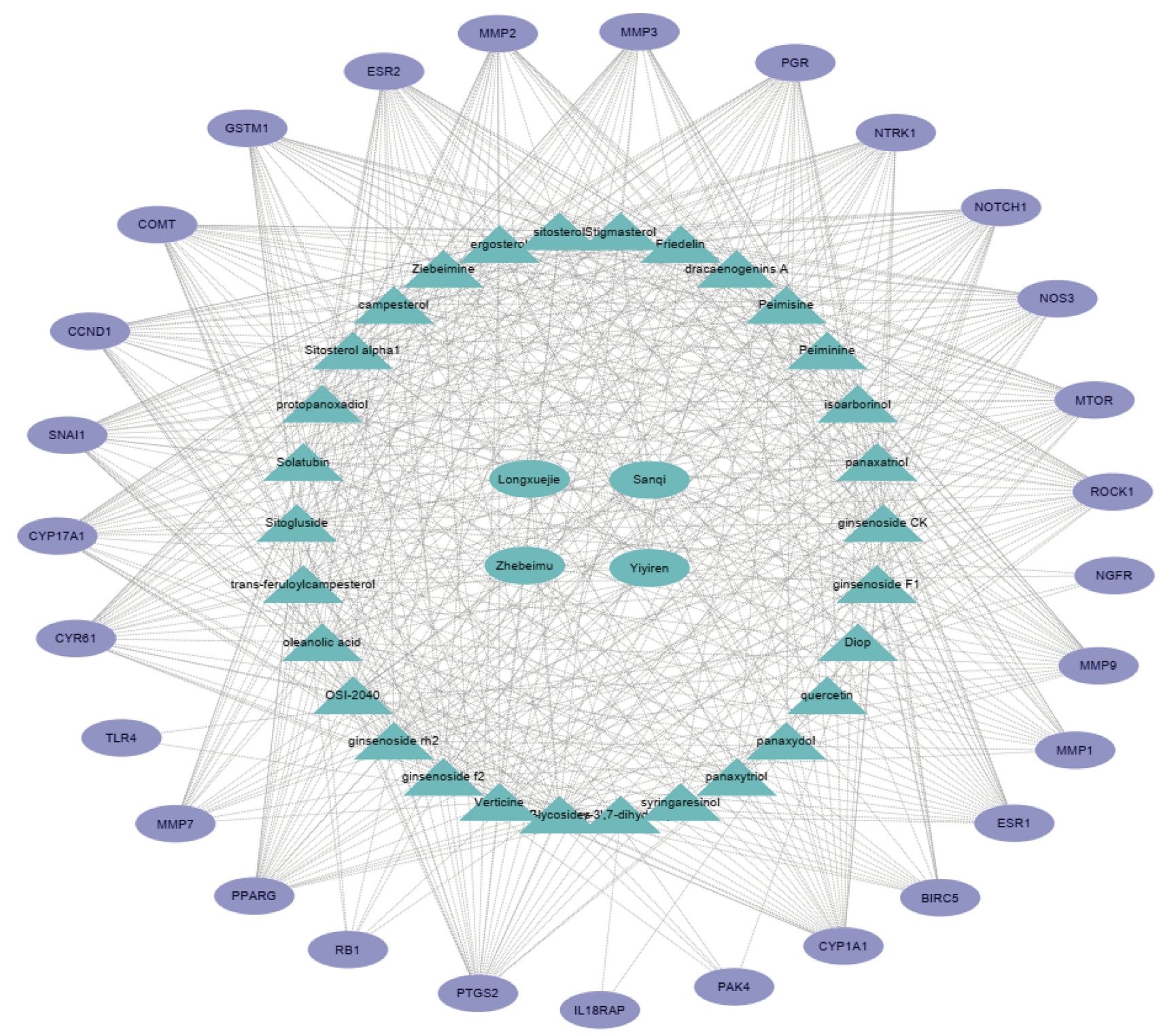

$\longrightarrow$ Drug $\longrightarrow$ Compound $\longrightarrow$ Target

Figure 5 Network of compound-target with strong binding activity and major distribution for SZC treating AM. It was constructed with 62 nodes (4 drugs, 30 compounds, and 28 targets) and 578 edges The blue triangles and purple ellipses represent crucial bioactive compounds of SZC and core corresponding targets of AM, respectively. The blue ellipses represent drugs in SZC. The gray edges represent the mutual relationships between targets and compounds.

weaker inhibition of NO than DXM $(\mathrm{P}<0.001)$, of which, notoginsenoside $\mathrm{R} 1$ got the lowest inhibition rate.

\section{The Cytokine Regulation of SZC}

The results shown in Figure 9A suggested that SZC inhibited IL-6 with no significant difference versus DXM and the compounds exhibited different strength, pterostilbene, 7,4'-dihydroxyflavone, and ginsenoside Rd inhibited IL-6 significantly stronger than DXM $(\mathrm{P}<0.01)$, of which, pterostilbene exhibited the strongest inhibition; notoginsenoside R1, ginsenoside Rg1, and ginsenoside $\mathrm{Rb} 1$ exhibited a significantly weaker inhibition than DXM $(\mathrm{P}<0.05)$ but were still more effective than resveratrol, loureirin A, loureirin $\mathrm{B}$, and peiminine.

SZC promoted the production of IL-10, but the compounds regulated IL-10 in two opposite directions. As shown in Figure 9B, resveratrol, pterostilbene, 7,4'dihydroxyflavone, loureirin $\mathrm{A}$, loureirin $\mathrm{B}$, and peiminine promoted the production of IL-10, while notoginsenoside $\mathrm{R} 1$, ginsenoside $\mathrm{Rg} 1$, ginsenoside $\mathrm{Rb} 1$, and ginsenoside $\mathrm{Rd}$ 
Table I The 30 Key Potential Active Compounds of SZC in Treating AM

\begin{tabular}{|c|c|c|}
\hline $\begin{array}{l}\text { PubChem } \\
\text { CID }\end{array}$ & Compound & Drug \\
\hline 3083151 & Ziebeimine & Zhebeimu \\
\hline 131900 & Verticine & Zhebeimu \\
\hline 443023 & Syringaresinol & Zhebeimu \\
\hline 65727 & Solatubin & Zhebeimu \\
\hline 222284 & Sitosterol & $\begin{array}{l}\text { Zhebeimu/Yiyiren/ } \\
\text { Sanqi }\end{array}$ \\
\hline 161294 & Peimisine & Zhebeimu \\
\hline $16769 \mid$ & Peiminine & Zhebeimu \\
\hline 6918328 & OSI-2040 & Zhebeimu \\
\hline |378659| & Trans-feruloylcampesterol & Yiyiren \\
\hline 5280794 & Stigmasterol & Yiyiren/Sanqi \\
\hline 9548595 & Sitosterol al & Yiyiren \\
\hline $12305 \mid 82$ & Isoarborinol & Yiyiren \\
\hline 91472 & Friedelin & Yiyiren \\
\hline 444679 & Ergosterol & Yiyiren \\
\hline 173183 & Campesterol & Yiyiren \\
\hline 5742590 & Sitogluside & Sanqi \\
\hline 5280804 & Quercetin & Sanqi \\
\hline 11213350 & Protopanoxadiol & Sanqi \\
\hline 93484 & Panaxytriol & Sanqi \\
\hline 5283280 & Panaxydol & Sanqi \\
\hline 73599 & Panaxatriol & Sanqi \\
\hline 10494 & Oleanolic acid & Sanqi \\
\hline II 9307 & Ginsenoside Rh2 & Sanqi \\
\hline 9852086 & Ginsenoside F2 & Sanqi \\
\hline 9809542 & Ginsenoside FI & Sanqi \\
\hline 9852086 & Ginsenoside CK & Sanqi \\
\hline 395120 & Diop & Sanqi \\
\hline 101389811 & Dracaenogenins A & Longxuejie \\
\hline 44259961 & $\begin{array}{l}\text { 4'-methoxy } \\
\text {-3',7-dihydroxyflavone }\end{array}$ & Longxuejie \\
\hline 637579 & Glycosides & Longxuejie \\
\hline
\end{tabular}

discouraged the production of IL-10. SZC exhibited the most extremely significant promotion of IL-10 versus LPS ( $\mathrm{P}<$ 0.001 ), followed by resveratrol, peiminine, and pterostilbene; nevertheless, notoginsenoside R1, ginsenoside Rg1, and ginsenoside Rd inhibited IL-10 with no significant difference from DXM. The raw array data of cell viability, NO, IL-6 and IL-10 is listed in Supplementary Table S5.

\section{Discussion}

\section{Active Compounds of SZC in Treating AM}

SZC is known to be composed of four TCMs, each medicine contains numerous compounds while the resin medicine Longxuejie could not be searched from any of the existing
Table 2 Information of 28 Core Potential Targets in SZC Treatment of AM

\begin{tabular}{|l|l|l|}
\hline $\begin{array}{l}\text { UniProt } \\
\text { CID }\end{array}$ & Protein Name & Gene \\
Name
\end{tabular}

TCM databases, the information of absorption, distribution, metabolism, and excretion (ADME) is insufficient, therefore 3D molecular structure docking was applied to completely screening of active compounds from the SZC compound database. It is worth noting that our finding of potentially active compounds were following the previous results, for instance, ginsenoside $\mathrm{Rh} 2$ has been proved to be active in preventing the growth of human ovarian cancer cells in vitro and in vivo, ${ }^{24}$ inhibiting the proliferation of MCF-7 cells through down-regulation of the expression level of cyclin $\mathrm{D},{ }^{25}$ and inhibiting angiogenesis in prostate cancer cells by decreasing VEGF and CNNM1. ${ }^{26}$ Elsewhere, peiminine has been reported to be a potential component that exhibited the ability of anti-inflammation. Further study has identified that 


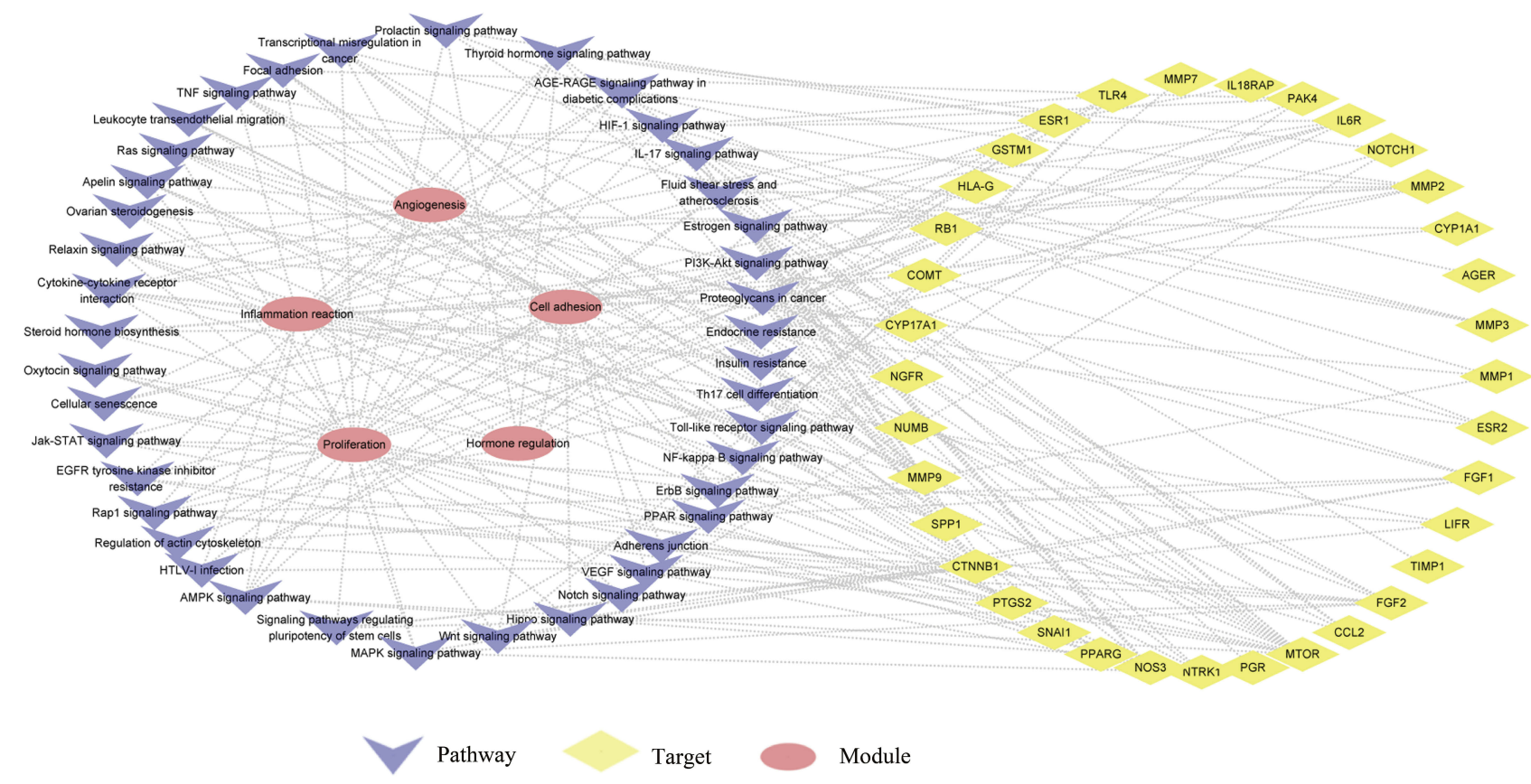

Figure 6 Target-pathway network of SZC treating AM. The purple arrows represent pathways connected with AM, the yellow rhombuses represent potential targets related to AM, and the pink ellipses represent regulatory modules of the pathways. The gray edges represent the mutual relationships between the targets and pathways.

peiminine regulated AKT/NF- $\mathrm{kB}$ p65, ERK1/2, and p38 signaling pathways in treating inflammatory mastitis induced by LPS in mice. ${ }^{27}$ It has also been found that peiminine inhibited the proliferation of colorectal cancer cells by regulating the $\mathrm{PI} 3 \mathrm{~K} / \mathrm{Akt} / \mathrm{mTOR}$ pathway and oxidative stress. ${ }^{28}$ Besides, estrogenic activities of 7,4'-dihydroxyflavone were discovered to be related to the properties of its structure. ${ }^{29}$ Stigmasterol can be found in three herbs of SZC exhibited diverse bioactivities in estrogenic effect, inflammation, antiproliferation, and angiogenesis. For instance, oxidation products of stigmasterol interfered with the female sex hormone $17 \beta$-estradiol in human breast and endometrium cells. ${ }^{30}$ Stigmasterol itself decreased inflammation by modulating the MAPK/NF- $\mathrm{KB}$ ROCK1 pathway, ${ }^{31}$ combined stigmasterols achieved more obvious anti-proliferation in Caco-2 cells than a single stigmasterol, ${ }^{32}$ and reduced TNF- $\mathrm{a}$ and VEGFR2 signaling by affecting phosphorylated Src, Akt, PCL, and FAK. ${ }^{33}$ It can be inferred that our approach is reliable and the screened active compounds act synergistically in the treatment of AM.

\section{Potential Targets in SZC Treatment of AM}

The multi-target therapeutic characteristics of TCM have become increasingly valued in revealing its mechanism, this could be expected to highlight the synergistic effects of TCM with the help of network techniques. ${ }^{34}$ From the enrichment of potential targets, it can be concluded that SZC mainly achieved the therapeutic efficacy to AM through regulating hormone regulation (ESR and PGR), inflammation reaction (PTGS2, NOS3, TLR4, and IL6R) and cell adhesion (Rho-associated coiled-coil containing protein kinase 1 [ROCK1], MMP), affecting cell proliferation (NOTCH1, peroxisome-proliferator activated receptor gamma [PPARG], snail family transcriptional repressor 1 [SNAL1]), and angiogenesis (cysteine-rich angiogenic inducer 61 [CYR61]).

Specifically, PTGS2 (COX-2) plays a multifunctional role and has a close connection with various diseases involving inflammation, immunization, cancer, and reproduction. ${ }^{35}$ For AM, it was explained that NF- $\mathrm{\kappa B}$ mediated PTGS2 and VEGF expression in endometrial stromal cells derived from the tissue of AM patients. ${ }^{36}$ Further study of gene polymorphism revealed that those who carried two A alleles were more likely suffer from $\mathrm{AM}$, Samodelkin has explained it to be a genetic variation of $\mathrm{G}$ to $\mathrm{A}$ at the -1195 locus in the promoter region of the PTGS2 gene that increases the risk of $\mathrm{AM}^{37}$ ROCK1 was demonstrated to play a major role in the progression of various types of cancer. Most significantly, the expression level was positively correlated with MMP9, which was associated with invasion and 


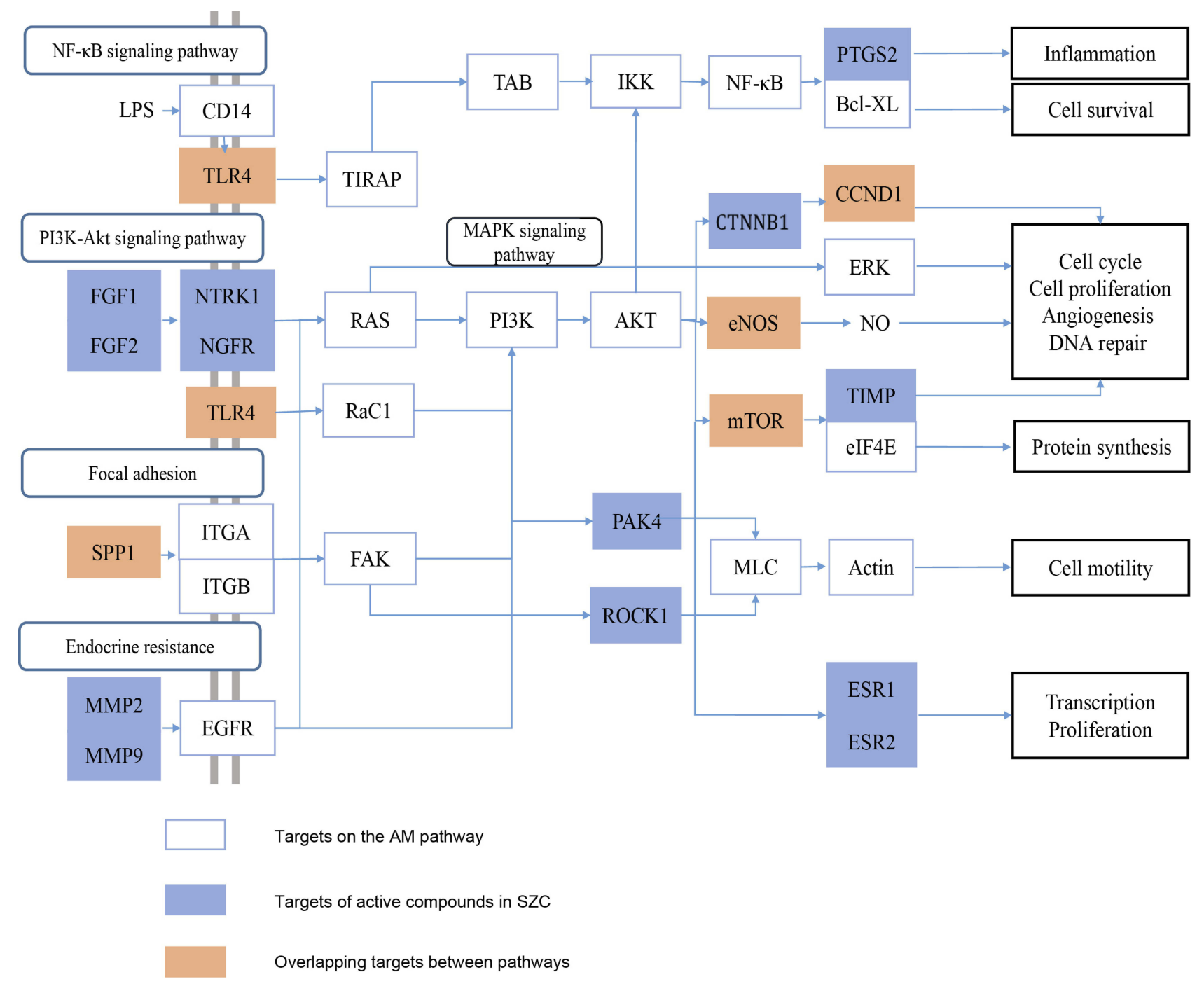

Figure 7 Confluence map of representative pathways. The white rectangles represent targets on the AM pathway, the orange rectangles represent overlapping targets of active compounds between pathways, and the blue rectangles represent targets of active compounds in SZC.

metastasis, and had become the key point of adhesion. ${ }^{38}$ Also, the overexpression of ROCK1 was proved to take part in dysmenorrhea and menstrual process in AM. ${ }^{39}$ As one of the single-pass transmembrane receptor protein family members, NOTCH1 was thought to play a critical role in the epithelial-mesenchymal transition affecting the proliferation of endometrial cells in the pathogenesis of AM for a higher expression of NOTCH1 in the proliferative phase. ${ }^{40}$ CYR61 has been identified to be one of the targets of the anti-angiogenic histone deacetylase 5 and was associated with a broad range of pathological processes such as impaired angiogenesis, fibrosis, and cancer on account of its ability to bind different combinations of co-receptors. The higher expression of CYR61 in ectopic endometrium than in eutopic endometrium might be involved in the pathogenesis of $\mathrm{AM}^{41}$

\section{Pathways in SZC Treatment of AM}

Close connection and intricate crosstalk were revealed by mapping the four pathways. It can be found that the PI3KAkt signaling pathway might be the major pathway as it involves the most predicted targets and the other three pathways. The PI3K-Akt signaling pathway is well known to play crucial roles in various cellular processes including proliferation, invasion, metastasis, angiogenesis, and apoptosis which are associated with inflammation regulation. Activation of the PI3K-Akt-eNOS signaling pathway helps to regulate angiogenesis through mediating cell proliferation and migration and leads to NO level and 


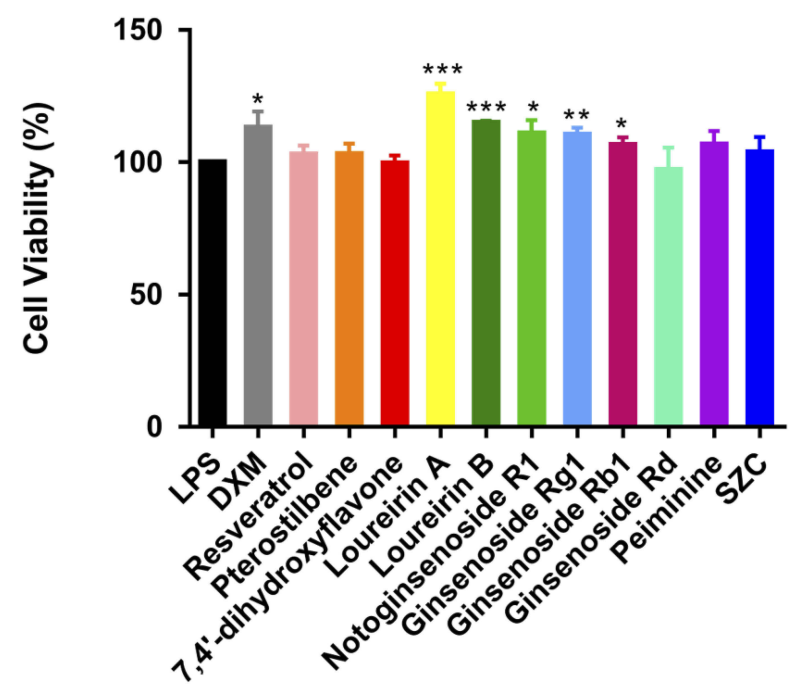

A

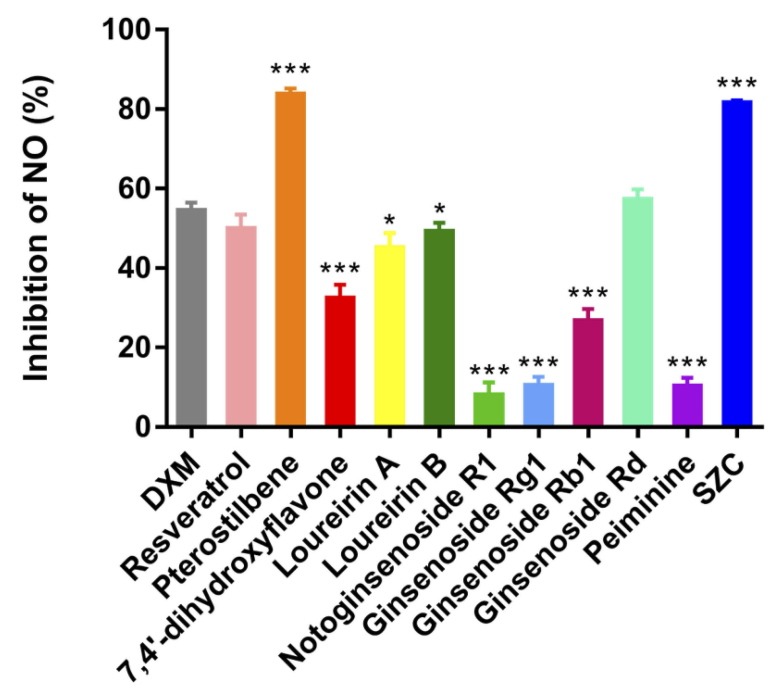

B

Figure 8 (A) Viability of LPS stimulated RAW264.7 cells after being treated by active compounds of SZC and (B) inhibition of synthesis of nitric oxide by active compounds of SZC at concentrations of $20 \mu \mathrm{M}$ (resveratrol, pterostilbene, 7,4'-dihydroxyflavone), $50 \mu \mathrm{M}$ (loureirin A, loureirin B, notoginsenoside RI, ginsenoside RgI, ginsenoside RbI, ginsenoside Rd, and peiminine), $50 \mu \mathrm{g} / \mathrm{mL}$ (SZC), and I $\mu \mathrm{M}(\mathrm{DXM})$ as positive control. Data were obtained from triplicate independent experiments and are presented as means $( \pm \mathrm{SD})$. $* \mathrm{P}<0.05$, **P $<0.01$, *** $\mathrm{P}<0.001$.

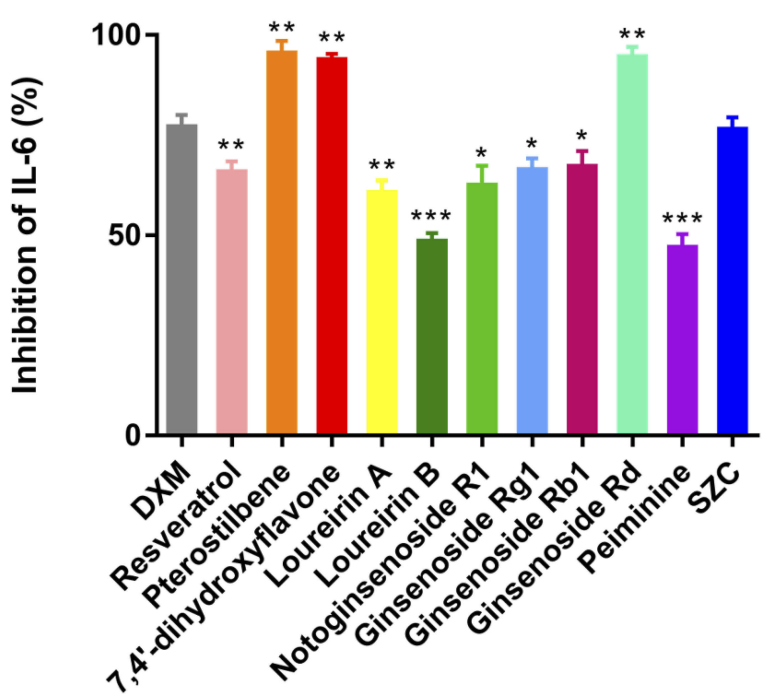

A

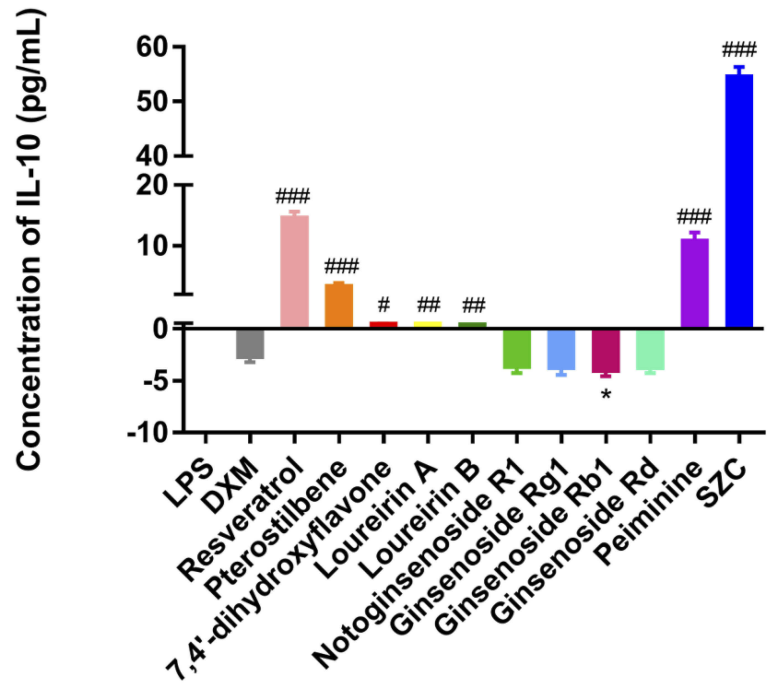

B

Figure 9 (A) Inhibition of releasing IL-6 by active compounds of SZC and (B) production of IL- I0 after being treated by active compounds of SZC at concentrations of 20 $\mu \mathrm{M}$ (resveratrol, pterostilbene, 7.4'-dihydroxyflavone), $50 \mu \mathrm{M}$ (loureirin $\mathrm{A}$, loureirin $\mathrm{B}$, notoginsenoside RI, ginsenoside RgI, ginsenoside RbI, ginsenoside Rd, and peiminine), $50 \mu g / \mathrm{mL}(\mathrm{SZC})$, and I $\mu \mathrm{M}(\mathrm{DXM})$ as positive control. Data were obtained from triplicate independent experiments and presented as means $( \pm \mathrm{SD})$. $* \mathrm{P}<$ 0.05 , **P $<0.01$, ***P $<0.001$ versus $\mathrm{DXM},{ }^{\#} \mathrm{P}<0.05,{ }^{\# \#} \mathrm{P}<0.01$, ${ }^{\# \#} \mathrm{P}<0.001$ versus LPS.

eNOS activity regulation. ${ }^{42}$ It has been demonstrated that PI3K-Akt is essential in decreasing inflammatory cytokines induced with LPS (IL-6, TNF-a, and IL-10), ${ }^{43,44}$ accompanied by a decrease in cell proliferation and migration. ${ }^{45}$ Additionally, the PI3K-Akt-mTOR (mammalian target of rapamycin) signaling pathway regulates cellular processes through the co-regulated targets with different functions. For example, cytoskeletal changes are associated with PI3K-AKT-mTOR signaling mediated alterations in focal adhesion kinase (FAK). Activated mTORC1 initiates pro-metastatic actin cytoskeleton remodeling by activating ROCK. $^{46}$ Moreover, it has been validated that the expression of phosphorylated mTOR was significantly higher in the ectopic endometrial 
than that in the eutopic endometria of AM patients, indicated that modulating the PI3K-Akt-mTOR signaling pathway to regulate inflammation might provide a promising therapeutic approach for $\mathrm{AM} .{ }^{47}$

As for the NF- $\kappa B$ signaling pathway, it is the canonical pathway broadly involved in regulating various physiological activities including immunity, inflammation, cell survival, and cell proliferation. The NF- $\kappa \mathrm{B}$ protein can be stimulated by a variety of agents like cytokines, chemokines, microbial and viral products, and DNA damage. The genes regulated by NF- $\kappa \mathrm{B}$ are highly associated with many other signaling pathways, and the outcome of NF$\kappa \mathrm{B}$ followed the diverse inductions of cells and special regulations of its target genes. ${ }^{48}$ For example, NF- $\mathrm{BB}$ signaling can be deregulated by microbial products and genetic alterations in $\mathrm{NF}-\kappa \mathrm{B}$ and other signaling pathway components in many human diseases, including cancers and chronic inflammation. ${ }^{49}$ In inflammatory responses, $\mathrm{NF}-\kappa \mathrm{B}$ plays an essential role in modulating the expression of pro-inflammatory cytokines. It was reported that the TLR4-mediated inflammatory response induced by LPS could be suppressed through the inhibition of iNOS and COX-2 expression and the decrease of $\mathrm{NO}$ and proinflammatory cytokines (including IL-1 $\beta$ and IL-6) by inhibiting the Akt-NF- $\kappa \mathrm{B}$ pathway. ${ }^{50}$ Besides, the NF- $\mathrm{BB}$ signaling pathway was examined to explain how the antiinflammatory effect was processed by reducing the production of $\mathrm{NO}$, the expression of iNOS and COX-2, and pro-inflammatory cytokines (including TNF-a, IL-1 $\beta$, IL6 , and MCP-1). ${ }^{51}$ It was also revealed that the anti-inflammatory activity could be exerted through the NF- $\kappa \mathrm{B}$ and PI3K/Akt pathways by influencing IL-10, IL-6, and TNF-a release, regulating iNOS, COX-2, NF- $\kappa \mathrm{B}, \mathrm{Akt}$, and p-Akt expression. ${ }^{52}$ Most of all, NF- $\kappa$ B plays a crucial role in the $\mathrm{NF}-\kappa \mathrm{B}$ signaling pathway, which was strongly suggested to be the potential target in the treatment of AM, at least in terms of relieving dysmenorrhea and menorrhagia. Not only does it regulate COX-2 but also the genes and their receptors encoding pro-inflammatory cytokines and chemokines, the expression of which has been reported to be related to inflammation in $\mathrm{AM} .^{53-55}$

Focal adhesion refers to the specialized structures formed at the cell-extracellular level, which are mediated by membrane receptors, actin-cytoskeleton, protein kinases, and phosphatases, playing an essential role in important biological processes including cell motility and proliferation. It was found focal adhesion took part in epithelial-mesenchymal transition associated with the pathogenesis of AM by regulating FAK, and in a laboratory investigation, results suggested the process might be associated with the PI3K-Akt pathway. ${ }^{56}$ Besides, the research revealed that secreted phosphoprotein 1 (SPP1) was the key protein expressed in specific phenotypes with different levels. ${ }^{57}$ Furthermore, the abnormal expression of SPP1 might cause infertility in some patients with $\mathrm{AM}^{58}$

Endocrine resistance is regarded as pathogenesis of hormone-dependent diseases. This pathway can be activated by an estrogen signaling pathway to express ER and is closely related to the expression of growth factors, especially the growth factors on the PI3K-Akt-mTOR pathway. It has been proved that upregulating growth factor receptor pathways might help cells escape estrogen or ER dependence through an alternative mechanism. ${ }^{59}$ Furthermore, experiments on the inhibition of the apoptotic effects of estrogen stimulation on ER+ cells suggested that a combined blockade of ER and PI3K might be the most effective. ${ }^{60}$ On the other hand, MMP-2 and MMP-9 mediated by EGFR are crucial for modulating invasion and metastasis because the regulation occurs through promoting PI3K. ${ }^{61}$ Additionally, Miller has discussed that activation of the Cyclin D-associated pathway could promote endocrine resistance, and treatment with the inhibitors could abrogate the proliferation of endocrine-resistant cells. ${ }^{62}$

\section{The Anti-Inflammatory Activity of SZC in vitro}

SZC exhibited the anti-inflammatory activity by the cooperation of multi-compound through inhibiting the release of NO and IL-6, and promoting the release of IL-10 in vitro. The compounds contributed differently to the SZC antiinflammatory activity of SZC, for instance, the flavonoids (resveratrol, pterostilbene, 7,4'-dihydroxyflavone, loureirin A, loureirin B) and the alkaloid (peiminine) exhibited the same trend in regulating NO, IL-6 and IL-10 regulation with SZC. While the saponins (notoginsenoside R1, ginsenoside $\mathrm{Rg} 1$, ginsenoside Rb1, ginsenoside Rd) exhibited the same trend in NO, IL-6, but the opposite trend with IL-10 versus SZC, eventually the IL-10 release promotion of flavonoids outweighed the inhibition of the saponins. As the antiinflammatory cytokine, the production of IL-10 counteracts the expression of IL-6, which is the pro-inflammatory cytokine during inflammation. ${ }^{63}$ Besides, the mechanism of the inflammatory response caused by overactivation of macrophages involves phenotypic changes of M1/M2-type macrophages, the pro-inflammatory cytokines such as IL-6, TNF-a, MCP-1, iNOS are produced by M1-type macrophages, and 
the anti-inflammatory cytokine including IL-4, IL-10, TGF$\beta 1$ are produced by M2-type macrophages. ${ }^{64,65}$ Our results revealed that both SZC and compounds could inhibit IL-6 production, while the flavonoids, alkaloid, and SZC promoted IL-10 production. It can be inferred that SZC performed the anti-inflammatory activity by inducing the M1 pro-inflammatory phenotype to polarize to the M2 antiinflammatory phenotype, and that might be contributed by the flavonoids and alkaloid of SZC. However, the M2 activation might be restrained by saponins downregulating the expression of M2 markers, such as CD206, IL-10, and YM-1 during the inflammation. ${ }^{66}$ As the saponins of SZC was found to reduce IL-10 production in our study, we deduced that the activation of macrophages toward M1 and M2 phenotype might coexist during the anti-inflammatory activity of SZC, more M1/M2 subtypes should be examined extensively in further studies.

In clinical research, Li has validated that IL-6 and IL-8 were over-expressed in the eutopic and ectopic endometrium of patients with AM. ${ }^{67}$ On the other hand, the expression of IL-10 was decreased in uteri of AM patients, that was supposed to be associated with impaired endometrium receptivity in $\mathrm{AM}$ patients. ${ }^{68}$ It also has been reported that NO, IL-10, IL-17A, IL-7, and MCP-1 were all found to possibly contribute to the pathogenesis of AM. ${ }^{69,70}$ As mentioned above, the NO level and inflammatory cytokines including IL-6, and IL-10 were closely related to the anti-inflammatory activity, might be decreased by suppressing the PI3K-Akt signaling pathway and NF- $\kappa \mathrm{B}$ signaling pathway. ${ }^{71,72}$ Therefore, it can be concluded that the anti-inflammatory activity of SZC is achieved by the synergetic action of the active compounds inhibiting the NO synthesis, IL-6 expression and the regulation of IL-10 expression through influencing the PI3KAkt signaling pathway and $\mathrm{NF}-\kappa \mathrm{B}$ signaling pathway.

\section{Conclusion}

In this study, a systematic network pharmacology analysis approach for exploring the mechanism of SZC in the treatment of AM was established and the anti-inflammatory activity of SZC was observed in vitro. The integrated approach automatically acquired the information and linked the docking platform, identified the potential active compounds and targets based on molecular docking combining with CTPG prediction, then implemented the PPI analysis, network construction and KEGG analysis, which provide insights into the biological activity of SZC. The results were as follows. First, 30 major compounds and 28 core targets were identified from the potential active ones. Second, five main therapeutic modules involving inflammation reaction, hormone regulation, cell adhesion, proliferation, and angiogenesis were collected. Third, four crucial intersected pathways including PI3K-Akt signaling pathway, NF- $\kappa \mathrm{B}$ signaling pathway, focal adhesion, and endocrine resistance were mapped, among them, the PI3KAkt signaling pathway and NF- $\mathrm{KB}$ signaling pathway were mainly related to the inflammation reaction module. Four, the anti-inflammation activity of SZC was the act by the cooperation of its multiple components regulating NO, IL6 , and IL-10, which was consistent with the analysis result. Overall, our findings indicated that SZC achieved the treatment of AM by influencing inflammation reaction, hormone regulation, cell adhesion, proliferation, and angiogenesis, furthermore, its anti-inflammatory activity was conducted by the multi-compound through inhibiting NO and IL-6, both inhibiting and promoting IL-10, which will provide rich clues for the further research on SZC in the treatment of AM. However, the ADME information and the content of compounds in SZC should be investigated in future research.

\section{Acknowledgments}

The authors would like to acknowledge the technical supports from De-Hui Du, Shanghai Key Laboratory of Trustworthy Computing and Software Engineering Institute, East China Normal University, Shanghai, China, and the prophase data provided from JiaChun Li, Jiangsu Kanion Pharmaceutical Co., Ltd, Jiangsu, China.

\section{Author Contributions}

All authors contributed to data analysis, drafting and revising the article, gave final approval of the version to be published, and agree to be accountable for all aspects of the work.

\section{Funding}

This work was supported by programs of the National Natural Science Foundation of China [grant numbers 81872981]; Technological Major Special Project of China (Grant number 2018ZX09201008-002); Program of Shanghai Academic/Technology Research Leader [grant number 18XD1403700]; Project of the Shanghai Municipal Commission of Health and Family Planning [grant number 2017YQ072 and 201740152]; Xinglin Young Talent Program (2017.10-2020.09) and the projects sponsored by the development fund for Shanghai talents [grant number 2018105]. 


\section{Disclosure}

Wei Xiao is employed by Jiangsu Kanion Pharmaceutical Co., Ltd, The authors report no other conflicts of interest in this work.

\section{References}

1. Hiniker SM, Donaldson SS. Recent advances in understanding and managing rhabdomyosarcoma. F1000Prime Rep. 2014;7:59.

2. García-Solares J, Donnez J, Donnez O, Dolmans MM. Pathogenesis of uterine adenomyosis: invagination or metaplasia? Fertil Steril. 2018;109(3):371-379. doi:10.1016/j.fertnstert.2017.12.030

3. TL R. The important roles of steroid sulfatase and sulfotransferases in gynecological diseases. Front Pharmacol. 2016;7(5 Pt 1):30. doi:10.3389/fphar.2016.00323

4. Leyendecker G, Wildt L. A new concept of endometriosis and adenomyosis: tissue injury and repair (TIAR). Horm Mol Biol Clin Investig. 2011;5(2):125-142. doi:10.1515/HMBCI.2011.002

5. Vannuccini S, Tosti C, Carmona F, et al. Pathogenesis of adenomyosis: an update on molecular mechanisms. Reprod Biomed Online. 2017;35(5):S1472648317302961. doi:10.1016/j.rbmo.2017.06.016

6. Shen MH, Liu XS, Zhang HQ, Guo SW. Transforming growth factor $\beta 1$ signaling coincides with epithelial-mesenchymal transition and fibroblast-to-myofibroblast transdifferentiation in the development of adenomyosis in mice. Hum Reprod. 2016;31(2):355-369. doi:10.1093/humrep/dev314

7. Chen XF, He XR, Liao XP. Clinical observation on 30 cases of adenomyosis dysmenorrhea treated with Chailing Xiaozheng decoction. J Qiqihar Med Coll. 2016;37(22):2792-2794.

8. Wang S, Luo MY, Yuan Y, et al. Effect of Xuanyu Tongjing Decoction in the treatment of dysmenorrhea of uterine adenomyosis. Contemp Chin Med. 2019;26(1):190-192.

9. Dan LQ. The clinical effect of Jiawei Siwu decoction in the treatment of adenomyosis. Electron J Clin Med Lit. 2018;5(96):135-138.

10. Wang HY, Bao XH, Dai JF. Treatment of ovarian cyst after ovulation-induction with Sanjie Zhentong capsule. Chin J Integr Trad West Med. 2008;28(28):1026-1028.

11. Zou J, Guan Z, Zhang WY, Xiao W, Li YL. Beneficial effects of the Chinese herbal medicine Sanjie Zhentong capsule on experimental endometriosis in rats. Chin $J$ Nat Med. 2013;11(6):666-672. doi:10.1016/S1875-5364(13)60077-1

12. Zou J, Guan Z, Zhang WY. Comparison of the effects of Sanjie Zhentong capsule and danazol on the endometriosis rats. Chin J Integr Trad West Med. 2012;32(8):1112-1116.

13. Chai HJ. Clinical observation of Sanjie Zhentong capsule in treating adenomyosis and infertility. North Pharm. 2017;14(9):68-69.

14. Shen XY, Yu LG. Therapeutic effect of Sanjie Zhentong capsule on dysmenorrhea caused by adenomyosis. J Clin Ration Drug Use. 2013;6(12):74.

15. Li SY, Li HY. Effects of Sanjie Zhentong capsule combined with indometin suppository on serum CA125, TNF-a, PGF2a in patients with adenomyosis. J Integr Trad Chin Wes Med. 2015;24(35):3954$3955+3980$.

16. Zhang R, Zhu X, Bai H, Ning K. Network pharmacology databases for traditional Chinese medicine: review and assessment. Front Pharmacol. 2019;10:1-14. doi:10.3389/fphar.2019.00001

17. Shi XQ, Yue SJ, Tang YP, et al. A network pharmacology approach to investigate the blood enriching mechanism of Danggui buxue Decoction. J Ethnopharmacol. 2019;235:227-242. doi:10.1016/j. jep.2019.01.027

18. Yang B, Wang N, Wang S, et al. Network-pharmacology-based identification of caveolin-1 as a key target of Oldenlandia diffusa to suppress breast cancer metastasis. Biomed Pharmacother. 2019;112:1-14. doi:10.1016/j.biopha.2019.108607
19. Hsin K-Y, Matsuoka Y, Asai Y, et al. systemsDock: a web server for network pharmacology-based prediction and analysis. Nucleic Acids Res. 2016;44:507-513. doi:10.1093/nar/gkw335

20. Clemente GP, Grassi R. Directed clustering in weighted networks: a new perspective. Chaos Solitons Fractals. 2017;107:26-38. doi:10.1016/j.chaos.2017.12.007

21. Guo SZ, Lu ZM, Zhe C, Hao L. Strength-strength and strength-degree correlation measures for directed weighted complex network analysis. IEICE Trans Inf Syst. 2011;94-D(11):2284-2287. doi:10.1587/transinf.E94.D.2284

22. Zhang GR, Yun WU, Sha WU, Xiao W. Simultaneous determination of five compounds in Sanjie Zhentong capsules by high-performance liquid chromatography coupled with ultraviolet and evaporative light scattering detectors. Chin J Exp Trad Med Formulae. 2015;21 (5):65-68.

23. Li J, Li D, Pan Y, et al. Simultaneous determination of ten bioactive constituents of Sanjie Zhentong capsule in rat plasma by ultra-highperformance liquid chromatography tandem mass spectrometry and its application to a pharmacokinetic study. $J$ Chromatogr $B$. 2017;1054:20-26. doi:10.1016/j.jchromb.2017.03.001

24. Nakata H, Kikuchi Y, Tode T, et al. Inhibitory effects of ginsenoside Rh2 on tumor growth in nude mice bearing human ovarian cancer cells. Cancer Sci. 2010;89(7):733-740.

25. Oh M, Choi YH, Choi S, et al. Anti-proliferating effects of ginsenoside Rh2 on MCF-7 human breast cancer cells. Int J Oncol. 1999;14 (5):869-875. doi:10.3892/ijo.14.5.869

26. Huang YQ, Huang HX, Han ZD, et al. Ginsenoside Rh2 inhibits angiogenesis in prostate cancer by targeting CNNM1. J Nanosci Nanotechnol. 2019;19(4):1942-1950. doi:10.1166/jnn.2019.16404

27. Li Y, Qian G, Wenjin G, et al. Farrerol relieve lipopolysaccharide (LPS)-induced mastitis by inhibiting AKT/NF- $\mathrm{BB}$ p65, ERK1/2 and P38 signaling pathway. Int J Mol Sci. 2018;19(6):1770. doi:10.3390/ ijms 19061770

28. Zheng Z, Xu L, Zhang S, et al. Peiminine inhibits colorectal cancer cell proliferation by inducing apoptosis and autophagy and modulating key metabolic pathways. Oncotarget. 2017;8(29):47619-47631. doi:10.18632/oncotarget.17411

29. Young CS, Tae Youl H, Yun AJ, et al. Estrogenic activities of isoflavones and flavones and their structure-activity relationships. Planta Med. 2008;74(1):25-32. doi:10.1055/s-2007-993760

30. Newill H, Loske R, Wagner J, et al. Oxidation products of stigmasterol interfere with the action of the female sex hormone 17beta-estradiol in cultured human breast and endometrium cell lines. Mol Nutr Food Res. 2007;51(7):888-898. doi:10.1002/ mnfr. 200700025

31. Lee S, Youn K, Jun M. Major compounds of red ginseng oil attenuate A $325-35$-induced neuronal apoptosis and inflammation by modulating MAPK/NF-кB pathway. Food Funct. 2018;9(8):4122-4134. doi:10.1039/C8FO00795K

32. Lopez-Garcia G, Cilla A, Barbera R, Alegria A. Antiproliferative effect of plant sterols at colonic concentrations on Caco-2 cells. J Funct Foods. 2017;39:84-90. doi:10.1016/j.jff.2017.10.006

33. Kangsamaksin $\mathrm{T}$, Chaithongyot $\mathrm{S}$, Wootthichairangsan $\mathrm{C}$, et al. Lupeol and stigmasterol suppress tumor angiogenesis and inhibit cholangiocarcinoma growth in mice via downregulation of tumor necrosis factor- $\alpha$. PLoS One. 2017;12(12):e0189628. doi:10.1371/ journal.pone. 0189628

34. Wang Y, Fan X, Qu H, Gao X, Cheng Y. Strategies and techniques for multi-component drug design from medicinal herbs and traditional Chinese medicine. Curr Top Med Chem. 2012;12(12):1356-1362. doi:10.2174/156802612801319034

35. Li B, Chen M, Liu X, Guo SW. Constitutive and tumor necrosis factor- $\alpha$-induced activation of nuclear factor- $\kappa \mathrm{B}$ in adenomyosis and its inhibition by andrographolide. Fertil Steril. 2013;100(2):568-577. doi:10.1016/j.fertnstert.2013.04.028 
36. Wang Y, Qu Y, Song W. Genetic variation in COX-2-1195 and the risk of endometriosis and adenomyosis. Clin Exp Obstetr Gynecol. 2015;42(2):168-172.

37. Samodelkin EI, Mercucheva NG, Kosareva PV, Nesterova LY. The role of prostaglandins and Cyclooxygenase in pathogenesis of chronic endometritis. Patol Fiziol Eksp Ter. 2017;61(2):98-100.

38. Luo D, Chen H, Li X, et al. Activation of the ROCK1/MMP-9 pathway is associated with the invasion and poor prognosis in papillary thyroid carcinoma. Int $J$ Oncol. 2017;51(4):1209-1218. doi:10.3892/ijo.2017.4100

39. Jiang C, Gong W, Chen R, et al. RhoA/ROCK/ARHGAP26 signaling in the eutopic and ectopic endometrium is involved in clinical characteristics of adenomyosis. J Int Med Res. 2018;46(12):5019-5029. doi: $10.1177 / 0300060518789038$

40. Qi S, Zhao X, Li M, et al. Aberrant expression of Notch1/numb/snail signaling, an epithelial mesenchymal transition related pathway, in adenomyosis. Reprod Biol Endocrinol. 2015;13(1):1-10. doi:10.1186/s12958-015-0084-2

41. Zhang D, Xia W, Li C, et al. Correlation between Cyr61 expression and clinicopathologic parameters in adenomyosis. J Reprod Immunol. 2016;118:42-49. doi:10.1016/j.jri.2016.08.004

42. Zheng ZZ, Liu ZX. Activation of the phosphatidylinositol 3-kinase/ protein kinase Akt pathway mediates CD151-induced endothelial cell proliferation and cell migration. Int J Biochem Cell Biol. 2007;39 (2):340-348. doi:10.1016/j.biocel.2006.09.001

43. Cianciulli A, Calvello R, Porro C, Trotta T, Salvatore R, Panaro MA. PI3k/Akt signalling pathway plays a crucial role in the anti-inflammatory effects of curcumin in LPS-activated microglia. Int Immunopharmacol. 2016;36:282-290. doi:10.1016/j. intimp.2016.05.007

44. Pengal RA, Ganesan LP, Wei G, Fang H, Ostrowski MC, Tridandapani S. Lipopolysaccharide-induced production of interleukin-10 is promoted by the serine/threonine kinase Akt. Mol Immunol. 2006;43(10):1557-1564. doi:10.1016/j. molimm.2005.09.022

45. Qiu L, Xu C, Jiang H, Li W, Tong S, Xia H. Cantharidin attenuates the proliferation and migration of vascular smooth muscle cells through suppressing inflammatory response. Biol Pharm Bull. 2018;42(1):34-42. doi:10.1248/bpb.b18-00462

46. Ersahin T, Tuncbag N, Cetin-Atalay R. The PI3K/AKT/mTOR interactive pathway. Mol Biosyst. 2015;11(7):1946-1954. doi:10.1039/ C5MB00101C

47. Guo JB, Gao JM, Yu XH. Expression of DJ-1 and mTOR in eutopic and ectopic endometria of patients with endometriosis and adenomyosis. Gynecol Obstet Invest. 2015;79(3):195-200. doi: $10.1159 / 000365569$

48. Perkins ND. Integrating cell-signalling pathways with NF- $\mathrm{KB}$ and IKK function. Nat Rev Mol Cell Biol. 2007;8(1):49-62. doi:10.1038/nrm2083

49. Wong ET, Tergaonkar V. Roles of NF-kB in health and disease: mechanisms and therapeutic potential. Clin Sci. 2009;116(6):451. doi: 10.1042/CS20080502

50. Lee YH, Jeon SJ, Kim SH, et al. A new synthetic chalcone derivative, 2-hydroxy-3',5,5'-trimethoxychalcone (DK-139), suppresses the Toll-like receptor 4-mediated inflammatory response through inhibition of the Akt/NF-kB pathway in BV2 microglial cells. Exp Mol Med. 2012;44(6):369-377. doi:10.3858/emm.2012.44.6.042

51. Kim JK, Jung DH, Kim SC, et al. Anti-inflammatory effect of Hemistepta lyrata Bunge (Bunge) on LPS- induced inflammation in RAW 264.7 cells. J Herbal Formula Sci. 2019;27(1):7-16.

52. Dai C, Zheng CQ, Meng FJ, Zhou Z, Jiang M. VSL\#3 probiotics exerts the anti-inflammatory activity via PI3k/Akt and;NF- $\kappa$ B pathway in rat model of DSS-induced colitis. Mol Cell Biochem. 2013;374(1-2):1-11. doi:10.1007/s11010-012-1488-3
53. Nie J, Lu YX. Immunoreactivity of progesterone receptor isoform $\mathrm{B}$, nuclear factor $\kappa \mathrm{B}$, and I $\kappa \mathrm{Balpha}$ in adenomyosis. Fertil Steril. 2009;92(3):886-889. doi:10.1016/j.fertnstert.2009.01.084

54. Wang F, Li H, Du X, Cui M, Wen Z. Expression of interleukin-10 in patients with adenomyosis. Fertil Steril. 2009;91(5):1681-1685. doi:10.1016/j.fertnstert.2008.02.164

55. Natalia S, Ioulia A, Anna M. Cytokine network of eutopic and ectopic endometrium in women with adenomyosis. Am J Reprod Immunol. 2015;47(4):251-255.

56. Zheng DX, Duan H, Wang S, Xu Q, Gan L, Dong QJ. FAK regulates epithelial-mesenchymal transition in adenomyosis. Mol Med Rep. 2018;18(6):5461-5472. doi:10.3892/mmr.2018.9600

57. Streuli I, Santulli P, Chouzenoux S, Chapron C, Batteux F. Serum osteopontin levels are decreased in focal adenomyosis. Reprod Sci. 2016;24(5):773-782. doi:10.1177/1933719116669054

58. Xiao Y, Li TC, Xia EL, Yang XL, Sun X, Zhou YF. Expression of integrin $\beta 3$ and osteopontin in the eutopic endometrium of adenomyosis during the implantation window. Eur $J$ Obstet Gynecol Reprod Biol. 2013;170(2):419-422. doi:10.1016/j. ejogrb.2013.05.007

59. Shira Peleg H, Tami R, Larysa R, Ido W. Endocrine resistance in breast cancer: focus on the phosphatidylinositol 3-kinase/akt/mammalian target of rapamycin signaling pathway. Breast Care. 2013;8 (4):248-255. doi:10.1159/000354757

60. Crowder RJ, Chanpheng P, Yu T, et al. PIK3CA and PIK3CB inhibition produce synthetic lethality when combined with estrogen deprivation in estrogen receptor-positive breast cancer. Cancer Res. 2009;69(9):3955. doi:10.1158/0008-5472.CAN-08-4450

61. Majumder A, Ray S, Banerji A. Epidermal growth factor receptor-mediated regulation of matrix metalloproteinase-2 and matrix metalloproteinase-9 in MCF-7 breast cancer cells. Mol Cell Biochem. 2018;452:1-11.

62. Miller TW. Endocrine resistance: what do we know?. Am Soc Clin Oncol Edu Book. 2013;2013:e37-e42.

63. Pranweerapaiboon K, Apisawetakan S, Nobsathian S, et al. An ethylacetate fraction of Holothuria scabra modulates inflammation in vitro through inhibiting the production of nitric oxide and proinflammatory cytokines via NF-kB and JNK pathways. Inflammopharmacology. 2019. doi:10.1007/s10787-019-00677-3

64. Chinetti-Gbaguidi G, Colin S, Staels B. Macrophage subsets in atherosclerosis. Nat Rev Cardiol. 2015;12:10-17. doi:10.1038/ nrcardio.2014.173

65. Geissmann F, Gordon S, Hume DA, Mowat AM, Randolph GJ. Unravelling mononuclear phagocyte heterogeneity. Nat Rev Immunol. 2010;10:453-460. doi:10.1038/nri2784

66. Kim B, Kim E, Lee E, et al. Panax notoginseng inhibits tumor growth through activating macrophage to M1 polarization. Am J Chin Med (Gard City N Y). 2018;46(06):1369-1385. doi:10.1142/ S0192415X18500726

67. Li C, Chen R, Jiang C, Chen L, Cheng Z. Correlation of LOX-5 and COX-2 expression with inflammatory pathology and clinical features of adenomyosis. Mol Med Rep. 2019;19(1):727-733. doi:10.3892/ mmr.2018.9618

68. Zhihong N, Yun F, Pinggui Z, et al. Cytokine profiling in the eutopic endometrium of adenomyosis during the implantation window after ovarian stimulation. Reprod Sci. 2016;23(1):124-133. doi:10.1177/ 1933719115597761

69. Liu JJ, Duan H, Wang S. Expression of nitric oxide in uterine junctional zone of patients with adenomyosis. Zhonghua Fu Chan Ke Za Zhi. 2013;48(7):504.

70. Zhi HZ, Su LZ, Zhang AJ. Cytokine profiling in the eutopic endometrium of adenomyosis during the implantation window after ovarian stimulation. Reprod Sci. 2016;23(1):124-133. doi:10.1177/ 1933719115597761 
71. Liu D, Cao G, Han L, Ye Y, Sima Y, Ge W. Flavonoids from radix Tetrastigmae inhibit TLR4/MD-2 mediated JNK and NF-אB pathway with anti-inflammatory properties. Cytokine. 2016;84:29-36. doi:10.1016/j.cyto.2015.08.003
72. Li JB, Wang HY, Yao Y, et al. Overexpression of microRNA-138 alleviates human coronary artery endothelial cell injury and inflammatory response by inhibiting the PI3K/Akt/eNOS pathway. $\mathrm{J} \mathrm{Cel}$ Mol Med. 2017;21(8):1482-1491. doi:10.1111/jcmm.13074

\section{Publish your work in this journal}

Drug Design, Development and Therapy is an international, peerreviewed open-access journal that spans the spectrum of drug design and development through to clinical applications. Clinical outcomes, patient safety, and programs for the development and effective, safe, and sustained use of medicines are a feature of the journal, which has also been accepted for indexing on PubMed Central. The manuscript management system is completely online and includes a very quick and fair peer-review system, which is all easy to use. Visit http://www. dovepress.com/testimonials.php to read real quotes from published authors.

Submit your manuscript here: https://www.dovepress.com/drug-design-development-and-therapy-journal 